\title{
SWC5.pdf
}

\section{Self-advanced propagation of light pulse in an optical fiber based on Brillouin scattering}

\author{
Sanghoon Chin, Miguel Gonzalez-Herraez*, Luc Thévenaz \\ Ecole Polytechnique Fédérale de Lausanne, STI-NAM Station 11, CH-1015 Lausanne, Switzerland \\ * Department of Electronics, University of Alcalá de Henares, E-28805 Madrid, Spain \\ sanghoon.chin@epfl.ch, miguelg@depeca.uah.es, luc.thevenaz@epfl.ch
}

\begin{abstract}
We propose a novel method to realize self-induced fast light and signal advancement with no distinct pump source in optical fibers, based on stimulated Brillouin scattering. This scheme can highly simplify real application systems.

(C)2007 Optical Society of America

OCIS codes: (060.4370) Nonlinear optics, fibers; (290.5900) Scattering, stimulated Brillouin; (350.5500) Propagation
\end{abstract}

\section{Introduction}

Dynamic control of the speed of light pulses propagating through optical media has received a great deal of recent interest because it opens up interesting opportunities to realize a timing tool for optical signal processing [1]. Particularly, the generation of fast light is challenging and fascinating for the scientific community since superluminal signal velocities can be achieved (however, preserving Einstein's causality). In all experiments of signal advancement a very large anomalous dispersion has to be induced in the medium at the signal frequency. Sharp atomic absorptions and electromagnetically induced absorption have provided means to create a large anomalous dispersion. In optical fibers, this condition can be readily produced by use of the narrow-band loss of stimulated Brillouin scattering (SBS) [3]. Furthermore, we developed a more sophisticated method that makes use of anomalous dispersion appearing between a gain-doublet created by SBS [4]. All these experiments, however, require a very strict control of the frequency spacing between the pump and the probe beams (normally this separation has to be exactly the Brillouin shift of the fiber used) and the states of polarization of the two beams should be parallel.

In this paper we propose a simple but efficient scheme to generate fast light in optical fibers, based on both spontaneous and stimulated Brillouin scattering. We demonstrate experimentally that a light pulse with a residual continuous-wave component propagating through an optical fiber as a signal can make itself speed up with no distinct pump laser. The high flexibility of Brillouin scattering gives us the possibility to realize a self-advanced signal. We believe that these results present a basis for a practical application in optical communication systems.

\section{Principle}

Stimulated Brillouin scattering is usually described as the interaction between two counter-propagating waves, a strong pump wave at $v_{\text {pump }}$ and a weak probe wave at $v_{\text {probe }}$. Under certain phase matching conditions, 


\section{SWC5.pdf}

$\left(v_{\text {pump }}=v_{\text {probe }}+v_{B}\right.$ or $\left.v_{\text {probe }}=v_{\text {pump }}+v_{B}\right)$, an acoustic wave is generated by electrostriction. When this acoustic wave is present, photons from the higher frequency wave are scattered to the lower frequency wave, leading to a narrowband amplification of the lower frequency wave, and to a narrowband loss in the higher frequency wave. As a consequence, the pump produces a narrow-band (30-50 MHz) gain/loss with peaks at the frequency of the probe signal. In the absence of a probe signal, spontaneous noise will seed the process. Above a certain power threshold, a significant part of the pump power will be transferred to a counter-propagating wave at $v_{\text {pump }}-v_{\mathrm{B}}$, that may in turn act as a pump to create a narrowband loss at $v_{\text {pump. }}$. In our experiment, a pulsed light signal plays simultaneously the role of pump and probe in the Brillouin interaction. When the signal power increases to reach the Brillouin threshold, it generates a strong backward-propagating Stokes wave whose frequency is downshifted by $\mathrm{v}_{\mathrm{B}}$ from that of the incident signal. Then this Stokes wave (which we will call pump hereafter) interferes with the incident wave to stimulate the Brillouin scattering process, and creates a spectral loss at the frequency of the probe. Note that this way the signal is accurately centered in the Brillouin loss regime since the frequency difference of the probe and the pump is given by the fiber itself and remains perfectly stable without any optical component or instrument such as a modulator or a microwave generator [5]. Moreover, in the case of having a large gain, the state of polarization of the pump precisely matches that of the probe signal, since the interaction transfers photons from the pump to the probe. These coherently transferred photons keep the polarization property of the pump, so that they drag the probe polarization state towards the pump polarization, resulting in a better polarization matching. [6].

\section{Experiments and Results}

A commercial DFB laser diode is used to generate a pulsed signal, operating at the wavelength of $1532 \mathrm{~nm}$. This beam passes through an isolator and is fast-optically gated with an external electro-optic modulator to produce a pulse train with duration of $50 \mathrm{~ns}$ (FWHM) at a repetition rate of $5 \mathrm{KHz}$. The repetition rate is chosen so that only one pulse is allowed to pass through the entire optical fiber to avoid any sort of interaction between adjacent pulses. The pulse includes certain DC power that is $14 \%$ of the peak power of the pulse. Then the pulse train power is boosted by a high gain erbium-doped fiber amplifier (EDFA) with 30dB gain and its power is controlled by a variable optical attenuator before entering a 12-km-long conventional dispersion shifted fiber (DSF). For this DSF we measured an SBS linewidth of $27 \mathrm{MHz}$ and a Brillouin shift of $10.6 \mathrm{GHz}$. By the principle described previously, when the signal power exceeds the Brillouin threshold, a strong Stokes wave is generated that in turn induces a narrowband loss at the signal frequency, and hence in these conditions, some advancement should be visible in the 


\section{SWC5.pdf}

signal at the fiber output.

Figure.1-(a) illustrates the measured advancement of the probe signal as a function of the input power,. A significant amount of advancement is achievable at powers above $10 \mathrm{dBm}$, which corresponds to the expected Brillouin threshold of the fiber, with a slope efficiency of 0.69ns/dBm. Figure.1-(b) shows the normalized time wave forms of the pulses for different probe powers after propagating through the DSF. It is seen that the pulse advancement increases with the probe power and the largest advancement achieved is $12 \mathrm{~ns}$ for a probe power of 27 $\mathrm{dBm}$. It must be pointed out that the signal pulse experiences nearly no distortion in all the experiments.
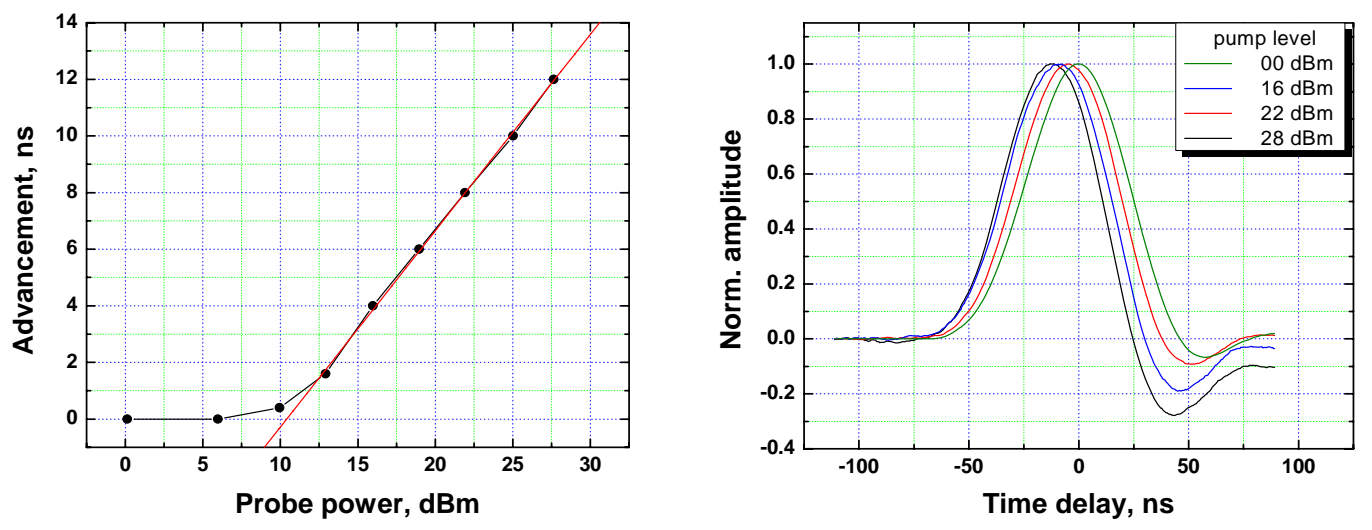

Fig.1. (a) The measured signal advancement as a function of the probe power. (b) The temporal traces of the probe pulses after propagating through the dispersion shifted fiber for different probe powers, showing clear pulse advancement.

\section{Conclusions}

We have demonstrated experimentally that self-advanced propagation of light pulses in optical fibers can be achieved, using both spontaneous and stimulated Brillouin scattering. From a practical point of view in real communication system, this scheme could be extremely convenient since the amount of advancement is simply controlled by the power of the incident signal, and no additional pump is needed.

\section{References}

1. R. W. Boyd and D.J. Gauthier, “'Slow' and 'Fast' light,” Ch. 6 in Progress in Optics 43, E. Wolf, Ed. (Elsevier, Amsterdam, 2002), 497-530.

2. K. Y. Song, M. Gonzalez Herráez and L. Thévenaz, Opt. Express 13, 82-88 (2005).

3. K. Y. Song, M. Gonzalez Herráez and L. Thévenaz, Opt. Express 13, 9758-9765 (2005).

4. M. Nikles, L. Thevenaz and P. A. Robert, J. Lightwave Technol. 15, 1842-1851 (1997)

5. M. O. Van Deventer and A. J. Boot, J. Lightwave Technol. 12, 585-590 (1994) 